\title{
Erratum to: Welfare Reforms, Child Welfare NGOs, and Accountability
}

\section{Erratum to:}

Chapter 3 in: J. Howell et al., NGOs and Accountability in China, https://doi.org/10.1007/978-3-319-90221-0_3

In the original version of the book, the sentence "Foreign NGOs Management Law 2016." in p. 79 of Chapter 3 has to be deleted, which is a belated correction. The erratum chapter have been updated with the change. 